\title{
Codebook Design and Hybrid Digital/AnalogCoding for Parallel Rayleigh Fading Channels
}

\author{
Shuying Shi, Erik G. Larsson and Mikael Skoglund
}

\section{Linköping University Pre Print}

N.B.: When citing this work, cite the original article.

C2011 IEEE. Personal use of this material is permitted. However, permission to reprint/republish this material for advertising or promotional purposes or for creating new collective works for resale or redistribution to servers or lists, or to reuse any copyrighted component of this work in other works must be obtained from the IEEE.

Shuying Shi, Erik G. Larsson and Mikael Skoglund, Codebook Design and Hybrid Digital/AnalogCoding for Parallel Rayleigh Fading Channels, 2011, accepted IEEE Transactions on Signal Processing.

Postprint available at: Linköping University Electronic Press http://urn.kb.se/resolve?urn=urn:nbn:se:liu:diva-69401 


\title{
Codebook Design and Hybrid Digital/Analog Coding for Parallel Rayleigh Fading Channels
}

\author{
Shuying Shi ${ }^{* 1}$, Erik G. Larsson ${ }^{1}$ and Mikael Skoglund ${ }^{2}$ \\ ${ }^{1}$ Linköping University, SE-581 83 Linköping, Sweden \\ ${ }^{2}$ Royal Institute of Technology (KTH), SE-100 44 Stockholm, Sweden
}

\begin{abstract}
Low-delay source-channel transmission over parallel fading channels is studied. In this scenario separate source and channel coding is in general highly suboptimal. A scheme based on hybrid digital/analog joint source-channel coding is therefore proposed, employing scalar quantization and polynomial-based analog bandwidth expansion. Simulations demonstrate substantial performance gains.
\end{abstract}

\section{Index Terms}

Hybrid digital/analog transmission, fading channels

\section{INTRODUCTION}

We consider the problem of transmitting a single analog (i.e., from a continuous alphabet) sample value over the Rayleigh fading channel. We study this problem in the case of bandwidth expansion, where a real-valued source sample $x$ is transmitted over $N$ parallel, independently fading channels. It is assumed that channel state information (CSI) is available at the receiver, but not at the transmitter. However, the noise power is possibly known at the transmitter.

There are essentially two approaches to the design of such a system: either one uses an analog modulation method or one uses a digital modulation method. With analog modulation, the received signal quality changes gradually with the channel SNR. Thus, analog methods perform well in the high-SNR region, but poorly in the in low SNR region. The design of analog bandwidth expansion systems for Gaussian channels was studied, e.g., in [1], [2].

Digital systems in general perform better than analog systems for low SNRs and can be designed to asymptotically achieve the theoretically optimal performance when transmitting infinitely long blocks and separating the source and channel coding. This will, however, introduce a delay into the system which may be undesirable. When CSI is unavailable at the transmitter, so that the transmission scheme cannot be adapted to the present channel state, digital systems suffer from the so-called "threshold" and "leveling-off" effects. If the channel quality goes below a certain threshold, the channel code will not be able to effectively protect against occasional bit errors and the system performance will rapidly deteriorate. On the other hand, if the channel quality is increased above a certain level, 
the system performance will not continue to improve but rather reach a constant level which is due to unrepairable errors introduced by the quantizer.

Generally, systems that mix digital and analog techniques can offer some advantages over pure digital and pure analog systems. In recent years, such hybrid digital/analog (HDA) systems have attracted increased interest [3], [4]. With HDA transmission, the system can achieve not only transmission with low delay, but also be made robust to channel variations in the absence of CSI at the transmitter. In [3], a class of HDA joint source-channel coding systems were proposed. These HDA systems can theoretically achieve the Shannon rate-distortion capacity limit at the designed channel SNR. Furthermore, they do not suffer from the leveling-off effect, although they do show a weakly pronounced threshold effect. A vector quantization-based HDA system was proposed in [4]. This system was based on long channel codes using a turbo error-correcting code to improve the performance at low channel SNRs.

In this paper, we consider HDA designs for the transmission of a single analog sample value over a parallel Rayleigh fading channel, with end-to-end mean-square-error (MSE) as the performance measure. This stands in contrast to most previous work on HDA transmission, which considered only transmission over the AWGN channel. The problem that we study is fundamental and merits a scientific investigation as such. It is also directly motivated by applications in which very small amounts of data must be reliably communicated over a highly unreliable channel under extremely tight latency constraints, for example in sensor networks, or for remote control. As such, our study has been mainly driven by the industrial applications identified in the EU-FP7 funded Low-Latency (LOLA) consortium, as discussed in more detail in [5]. Moreover, the HDA scheme that we propose can be used as a building block constituting an "inner code" in the physical-layer architecture for a complete communication link, in a similar manner as signal space diversity techniques like space-time block codes can be used as inner codes in multicarrier or multiantenna transmission schemes. Our paper presents two specific contributions:

1) We investigate the quantization and codebook design for the transmission of a single scalar value over parallel Rayleigh fading channels. We derive an upper bound on the overall end-to-end MSE distortion. By minimizing this bound, we obtain a codebook containing very short codes for transmission. Significant gains over other heuristic codebooks are observed in the simulations.

2) We present a low-delay HDA scheme obtained by combining this codebook design with the polynomial coding of [2]. Unlike [4], in our proposed scheme we do not employ long error correcting codes in the digital part, in order to avoid incurring additional delay and complexity.

The rest of this paper is organized as follows. In Section II, the polynomial coding scheme of [2] is reviewed. The codebook design for the Rayleigh fading channel is investigated in Section III. The proposed HDA scheme is presented in Section IV. Section V contains simulation results and Section VI concludes the paper.

\section{Review of the Polynomial Coding of [2]}

We first review the polynomial coding scheme of [2], which will be a building block in our proposed HDA design in Section IV. Consider the analog source-channel bandwidth expansion scheme illustrated in Fig. 1. The real-valued, analog source $x$ with pdf $p(x)$ is encoded by the function

$$
\boldsymbol{s}(x) \triangleq\left[s_{1}(x), \ldots, s_{N}(x)\right]^{T},
$$




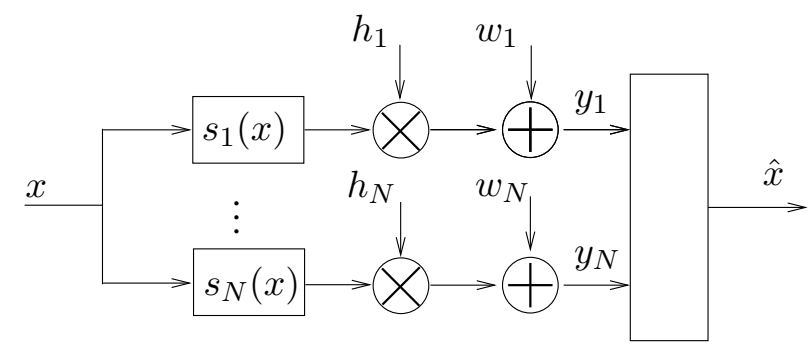

Fig. 1. Polynomial Coding

and transmitted over $N$ independent Rayleigh-fading channels (with real-valued coefficients). The diagonal matrix $\boldsymbol{H}=\operatorname{diag}\left\{\left[h_{1}, \ldots, h_{N}\right]\right\}$ contains the channel coefficients. The transmit power for each channel is assumed to be limited to $\rho$, i.e.,

$$
E\left[s_{n}(x)^{2}\right]=\int_{-\infty}^{\infty} p(x) s_{n}(x)^{2} d x \leq \rho, \quad \forall n \in\{1,2, \ldots, N\}
$$

The encoding functions $s_{n}(x), \forall n$, are chosen based on orthogonal polynomials [2]. In the case that the source sample $x$ is uniformly distributed over the interval $[-1,-1]$, i.e., $x \sim \mathcal{U}(-1,1)$, the orthogonal polynomials for $x \sim \mathcal{U}(-1,1)$ are known as the Legendre polynomials (see e.g., [6]). The Legendre polynomials, denoted here by $L_{m}(x), \forall m=0,1, \ldots, \infty$, are defined via the recurrence formula

$$
\begin{aligned}
& L_{0}(x)=1 \\
& L_{1}(x)=x \\
& L_{2}(x)=\frac{1}{2}\left(3 x^{2}-1\right) \\
& \vdots \\
& (m+1) L_{m+1}(x)=(2 m+1) x L_{m}(x)-m L_{m-1}(x) .
\end{aligned}
$$

We know that

$$
\int_{-1}^{1} L_{i}(x) L_{j}(x) d x=\frac{2}{2 m+1} \delta_{i j}
$$

where $\delta_{i j}$ is Kronecker's delta symbol. Thus, the corresponding orthonormal polynomials w.r.t. the weighting function $p(x)=1 / 2($ the pdf of $x \sim \mathcal{U}(-1,1)$ ) can be written as

$$
l_{m}(x) \triangleq \sqrt{2 n+1} L_{m}(x), \quad \forall m
$$

Given a set $\mathcal{I}=\left\{i_{1}, i_{2}, \ldots, i_{N}\right\}$ (the choice of $\mathcal{I}$ is discussed in [2]), the analog source-channel code $\boldsymbol{s}_{\mathcal{I}}(x)$ is generated by choosing

$$
\begin{aligned}
& s_{1}(x)=\sqrt{\rho} l_{i_{1}}(x) \\
& s_{2}(x)=\sqrt{\rho} l_{i_{2}}(x) \\
& \vdots \\
& s_{N}(x)=\sqrt{\rho} l_{i_{N}}(x) .
\end{aligned}
$$




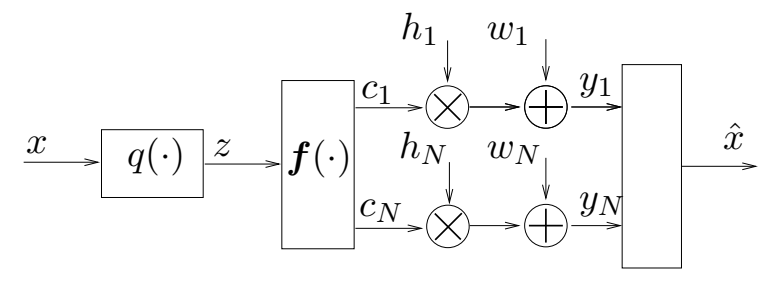

Fig. 2. Digital Transmission

Clearly, the power constraint in (1) is fulfilled:

$$
E\left[s_{n}(x)^{2}\right]=\frac{1}{2} \int_{-1}^{1}\left[\sqrt{\rho} l_{i_{n}}(x)\right]^{2}=\rho, \forall n .
$$

At the receiver, the observed vector $\boldsymbol{y}=\left[y_{1}, \ldots, y_{N}\right]^{T}$ is

$$
\boldsymbol{y}=\boldsymbol{H} \boldsymbol{s}_{\mathcal{I}}(x)+\boldsymbol{w}
$$

where $\boldsymbol{w}=\left[w_{1}, \ldots, w_{N}\right]^{T}$ is a vector of additive white Gaussian noise. The maximum-likelihood (ML) estimate of $x$ is

$$
\begin{aligned}
\hat{x} & =\arg \min _{x}\left\|\boldsymbol{H} \boldsymbol{s}_{\mathcal{I}}(x)-\boldsymbol{y}\right\|^{2} \\
& =\arg \min _{x} \sum_{i_{n} \in \mathcal{I}}\left(h_{n} \sqrt{\rho} l_{i_{n}}(x)-y_{n}\right)^{2} .
\end{aligned}
$$

The decoding procedure is equivalent to minimizing a polynomial of degree $2 i_{N}\left(i_{N}\right.$ is the largest index in $\left.\mathcal{I}\right)$. Efficient algorithms exist for minimizing univariate polynomials which makes this feasible, see e.g., [7].

\section{Codebook Design for Digital Transmission over PARAllel RAYleigh Fading Channels}

Now, consider the case that the source $x$ is encoded by means of a scalar quantizer, see Fig. 2 . The quantizer is a mapping $q(x)$, which to each $x$ assigns a quantized value $z$, drawn from a finite codebook $\mathcal{Z}=\left\{z_{1}, z_{2}, \ldots, z_{M}\right\}$, where $M$ is the number of quantization levels. The quantizer is completely described by the quantization codebook $\mathcal{Z}$ and the corresponding partitioning set $\Omega=\left\{\Omega_{1}, \Omega_{2}, \ldots, \Omega_{M}\right\}$. That is, the mapping $q(\cdot)$ is defined as

$$
q(x)=z_{i}, \text { if } x \in \Omega_{i}, \forall i \in\{1,2, \ldots, M\}
$$

The output of the quantizer is then mapped to a codeword $c=\left[c_{1}, \ldots, c_{N}\right]^{T}$ for transmission, where $c$ is chosen from a codebook $\mathcal{C}=\left\{\boldsymbol{c}_{1}, \ldots, \boldsymbol{c}_{M}\right\}$. We denote this one-to-one mapping by

$$
\boldsymbol{c}_{i}=\boldsymbol{f}\left(z_{i}\right), \forall i \in\{1,2, \ldots, M\}
$$

The overall MSE distortion caused by the quantizer and the channel can be computed as

$$
D(q ; \boldsymbol{f}) \triangleq \sum_{i=1}^{M} \sum_{j=1}^{M} P(j \mid i) \int_{\Omega_{i}} p(x)\left(x-z_{j}\right)^{2} d x,
$$

where $P(j \mid i) \triangleq P\left(\boldsymbol{c}_{j} \mid \boldsymbol{c}_{i}\right)$ denotes the pairwise error probability that $\boldsymbol{c}_{j}$ is received given that $\boldsymbol{c}_{i}$ is transmitted. If $z_{i}, \forall i$, are chosen to be the centroids of their respective encoding regions $\Omega_{i}, \forall i$, the overall distortion (5) reduces 
to the sum [8] of the source encoder distortion

$$
D_{s}(q) \triangleq \sum_{i=1}^{M} \int_{\Omega_{i}} p(x)\left(x-z_{i}\right)^{2} d x
$$

and the channel distortion

$$
D_{c}(\boldsymbol{f}) \triangleq \sum_{i=1}^{M} \sum_{j=1}^{M} P\left(\boldsymbol{c}_{i}\right) P(j \mid i)\left(z_{i}-z_{j}\right)^{2} .
$$

where $P\left(\boldsymbol{c}_{i}\right)$ is the a priori probability of the codeword $\boldsymbol{c}_{i}$. That is,

$$
D(q ; \boldsymbol{f})=D_{s}(q)+D_{c}(\boldsymbol{f}) .
$$

This implies that the quantizer $q(\cdot)$ and the mapping $f(\cdot)$ can be optimized separately.

For a given number of quantization levels $M$, the optimal quantizer in the sense of minimum $D_{s}(q)$ for a uniformly distributed source is the uniform quantizer. For example, if $M=4$, the quantization codebook is given by $\mathcal{Z}=\left\{z_{1}=-\frac{3}{4}, z_{2}=-\frac{1}{4}, z_{3}=\frac{1}{4}, z_{4}=\frac{3}{4}\right\}$.

In order to find the optimal mapping $\boldsymbol{f}(\cdot)$, we need to consider the channel distortion $D_{c}(\boldsymbol{f})$ in $(7)$. Given the received signal $\boldsymbol{y}=\boldsymbol{H} \boldsymbol{c}+\boldsymbol{w}$ and conditioned on the channel $\boldsymbol{H}$, the pairwise error probability ${ }^{1}$ is

$$
\begin{aligned}
P\left(\boldsymbol{c}_{i} \rightarrow \boldsymbol{c}_{j} \mid h_{1}, \ldots, h_{N}\right) & =Q\left(\sqrt{\frac{\left\|\boldsymbol{H} \boldsymbol{c}_{i}-\boldsymbol{H} \boldsymbol{c}_{j}\right\|^{2}}{2 \sigma^{2}}}\right) \\
& =Q\left(\sqrt{\frac{\sum_{n=1}^{N} h_{n}^{2}\left(c_{i, n}-c_{j, n}\right)^{2}}{2 \sigma^{2}}}\right),
\end{aligned}
$$

where $Q(\cdot)$ is the Q-function, $\sigma^{2}$ is the noise power and $c_{i, n}$ is the $n$th element of $\boldsymbol{c}_{i}$. Using the upper bound $Q(x) \leq \exp \left(-x^{2} / 2\right)$, for $x>0$, we get

$$
P\left(\boldsymbol{c}_{i} \rightarrow \boldsymbol{c}_{j} \mid h_{1}, \ldots, h_{N}\right) \leq \exp \left(-\frac{1}{4 \sigma^{2}} \sum_{n=1}^{N} h_{n}^{2}\left(c_{i, n}-c_{j, n}\right)^{2}\right) .
$$

Averaging w.r.t. $h_{1}, \ldots, h_{N}$ under the independent Rayleigh fading assumption, we have

$$
\begin{aligned}
P\left(\boldsymbol{c}_{i} \rightarrow \boldsymbol{c}_{j}\right) & \leq E_{h_{1}, \ldots, h_{N}}\left[\exp \left(-\frac{1}{4 \sigma^{2}} \sum_{n=1}^{N} h_{n}^{2}\left(c_{i, n}-c_{j, n}\right)^{2}\right)\right] \\
& =\prod_{n=1}^{N}\left(\frac{1}{1+\frac{1}{4 \sigma^{2}}\left(c_{i, n}-c_{j, n}\right)^{2}}\right) .
\end{aligned}
$$

Thus, using the expression in (9) for the pairwise error probabilities and the union bound to bound the terms $P(j \mid i)$ in (7), we obtain the following upper bound of the channel distortion

$$
D_{c}(\boldsymbol{f}) \leq \sum_{i=1}^{M} \sum_{j=1}^{M} P\left(z_{i}\right)\left(z_{i}-z_{j}\right)^{2} \prod_{n=1}^{N}\left(\frac{1}{1+\frac{1}{4 \sigma^{2}}\left(c_{i, n}-c_{j, n}\right)^{2}}\right) .
$$

\footnotetext{
${ }^{1}$ Here the expression in (9) is the traditional pairwise error probability with only two alternatives, while for (5) and (7) to be valid with equality, the expression $P(j \mid i)$ needs to take into account all alternatives.
} 
TABLE I

Optimized Codebook $\mathcal{C}$ For the RAyleigh FAding Channel, $\sigma^{2}=0.01, M=N=4, \rho=1$

\begin{tabular}{|crrr|}
\hline $\boldsymbol{c}_{1}$ & \multicolumn{1}{c}{$\boldsymbol{c}_{2}$} & \multicolumn{1}{c}{$\boldsymbol{c}_{3}$} & \multicolumn{1}{c|}{$\boldsymbol{c}_{4}$} \\
0.5022 & -0.4946 & -1.3273 & 1.3197 \\
0.3598 & 1.4177 & -0.5139 & -1.2636 \\
1.3787 & -0.4269 & 0.3796 & -1.3314 \\
1.4005 & 0.3931 & -1.2679 & -0.5257 \\
\hline
\end{tabular}

We can find a "good" codebook $\mathcal{C}$ by minimizing the upper bound (12). Since the mapping from $z_{i}$ to $\boldsymbol{c}_{i}$ is a one-to-one mapping, we have $P\left(\boldsymbol{c}_{i}\right)=P\left(z_{i}\right), \forall i$. To fulfill the power constraint per channel use, we require $\sum_{i=1}^{M} P\left(\boldsymbol{c}_{i}\right) c_{i, n}^{2}=\sum_{i=1}^{M} P\left(z_{i}\right) c_{i, n}^{2} \leq \rho$. Since $z_{i}-z_{i}=0$ and $\left(z_{i}-z_{j}\right)^{2}=\left(z_{j}-z_{i}\right)^{2}$, the optimization of the codebook $\mathcal{C}$ is equivalent to minimizing

$$
\begin{aligned}
\min _{\boldsymbol{c}_{1}, \ldots, \boldsymbol{c}_{M}} & \sum_{i=1}^{M} \sum_{j=i+1}^{M} P\left(z_{i}\right)\left(z_{i}-z_{j}\right)^{2} \prod_{n=1}^{N}\left(\frac{1}{1+\frac{1}{4 \sigma^{2}}\left(c_{i, n}-c_{j, n}\right)^{2}}\right) \\
\text { s.t. } & \sum_{i=1}^{M} P\left(z_{i}\right) c_{i, n}^{2} \leq \rho, \forall n=1, \ldots, N .
\end{aligned}
$$

The optimization problem (13) is non-convex and there exist multiple local optima. Even the global optimum is not unique. For example, in the case that $x \sim \mathcal{U}(-1,1), M=2, N=2$, global minimizers of (13) are $\boldsymbol{c}_{1}=[-\sqrt{\rho}-\sqrt{\rho}]^{T}, \boldsymbol{c}_{2}=[\sqrt{\rho} \sqrt{\rho}]^{T}$ and $\boldsymbol{c}_{1}=[\sqrt{\rho} \sqrt{\rho}]^{T}, \boldsymbol{c}_{2}=[-\sqrt{\rho}-\sqrt{\rho}]^{T}$.

It should be emphasized that to optimize the codebook according to (13) does not require us to know the channel $\boldsymbol{H}$, but the noise power. This optimization can be done offline, i.e., the codebook $\mathcal{C}$ can be created for different noise powers and stored at both the transmitter and the receiver. One possible way is to use local methods, like a gradient projection method, or an interior-point method, to find a local optimum of (13). With different initializations, we can get different local optima. The codewords can be chosen as the ones which yield the smallest objective value. The best codebook found, using a local search around 1000 randomly chosen initializations for $N=M=4$, $\sigma^{2}=0.01, \rho=1$ is given in Table I.

Remark 1. For the AWGN channel, it is easy to see from (9) that we can solve

$$
\begin{aligned}
\min _{\boldsymbol{c}_{1}, \ldots, \boldsymbol{c}_{M}} & \sum_{i=1}^{M} \sum_{j=i+1}^{M} P\left(z_{i}\right)\left(z_{i}-z_{j}\right)^{2} \cdot Q\left(\sqrt{\frac{\sum_{n=1}^{N}\left(c_{i, n}-c_{j, n}\right)^{2}}{2 \sigma^{2}}}\right) \\
\text { s.t. } & \sum_{i=1}^{M} P\left(z_{i}\right) c_{i, n}^{2} \leq \rho, \forall n=1, \ldots, N .
\end{aligned}
$$

to find the optimal codewords $\boldsymbol{c}_{i}, \forall i$. However, this optimization is numerically difficult. The reason is that the Q-function in (14) converges to zero much faster than what the RHS of (11) goes to zero when the noise power decreases. In our simulations, we have observed that the codebooks created for the fading channel are good for the AWGN channel as well (see Section V).

Generally, by increasing $M$, for a fixed quantizer $q, D_{s}(M)$ decreases. However, it is difficult to say whether $D_{c}(M, \boldsymbol{f})$ decreases or not, because it also depends on the mapping $\boldsymbol{f}(\cdot)$ and on the length of the codeword $N$. Joint optimization of $M$ and the codebook is not straightforward and out of scope of this paper. 


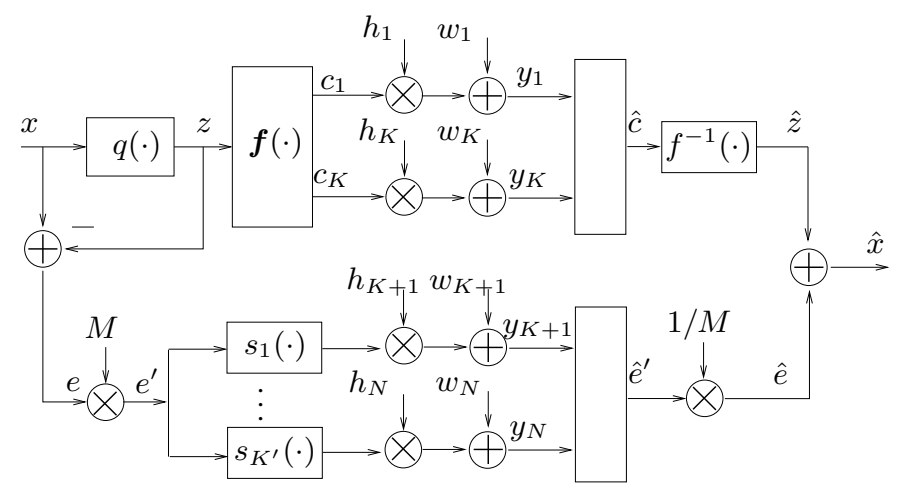

Fig. 3. Proposed Hybrid Digital/Analog Scheme

For a given quantizer $q(\cdot)$ and the mapping $\boldsymbol{f}(\cdot)$, the optimal decoder, in the minimum MSE sense, is given as

$$
\hat{x} \triangleq E[x \mid \boldsymbol{y}]=\int_{-\infty}^{\infty} x P(x \mid \boldsymbol{y}) d x
$$

Clearly,

$$
P(x \mid \boldsymbol{y})=\frac{P(\boldsymbol{y} \mid x) p(x)}{P(\boldsymbol{y})}
$$

where

$$
\begin{aligned}
P(\boldsymbol{y}) & =\sum_{i=1}^{M} P\left(\boldsymbol{c}_{i}\right) P\left(\boldsymbol{y} \mid \boldsymbol{c}_{i}\right) \\
& =\frac{1}{\sqrt{2 \pi \sigma^{2}}} \sum_{i=1}^{M} \exp \left(-\frac{1}{2 \sigma^{2}}\left\|\boldsymbol{y}-\boldsymbol{H} \boldsymbol{c}_{i}\right\|^{2}\right) \cdot P\left(\boldsymbol{c}_{i}\right) .
\end{aligned}
$$

Note that $P(\boldsymbol{y} \mid x)=\left.P(\boldsymbol{y} \mid \boldsymbol{c})\right|_{\boldsymbol{c}=\boldsymbol{f}(q(x))}$. Hence

$$
\begin{aligned}
\hat{x} & \triangleq E[x \mid \boldsymbol{y}]=\int_{-\infty}^{\infty} x P(x \mid \boldsymbol{y}) d x \\
& =\left.\frac{1}{P(\boldsymbol{y})} \int_{-\infty}^{\infty} x p(x) P(\boldsymbol{y} \mid \boldsymbol{c})\right|_{\boldsymbol{c}=\boldsymbol{f}(q(x))} d x \\
& =\frac{1}{P(\boldsymbol{y}) \sqrt{2 \pi \sigma^{2}}} \sum_{i=1}^{M} \int_{\Omega_{i}} x p(x) \exp \left(-\frac{1}{2 \sigma^{2}}\left\|\boldsymbol{y}-\boldsymbol{H} \boldsymbol{c}_{i}\right\|^{2}\right) d x \\
& =\frac{\sum_{i=1}^{M} \exp \left(-\frac{1}{2 \sigma^{2}}\left\|\boldsymbol{y}-\boldsymbol{H} \boldsymbol{c}_{i}\right\|^{2}\right) \int_{\Omega_{i}} x p(x) d x}{\sum_{i=1}^{M} \exp \left(-\frac{1}{2 \sigma^{2}}\left\|\boldsymbol{y}-\boldsymbol{H} \boldsymbol{c}_{i}\right\|^{2}\right) \int_{\Omega_{i}} p(x) d x} .
\end{aligned}
$$

Remark 2. Based on the ML detector we obtain with relative ease a criterion that can be used to optimize the codebook. Notwithstanding that, we want the best estimation performance in the MSE sense. Therefore we use the MMSE estimator (15) here.

\section{Proposed Hybid Digital/Analog System}

The proposed HDA system consists of a digital and an analog part that operate in parallel. The digital part is used to transmit the quantized value $z=q(x) \in \mathcal{Z}$, using $K$ channel uses. The analog part is used to send the quantization error $e=z-x$, using $K^{\prime} \triangleq N-K$ channel uses. Jointly optimizing the digital and analog parts of 
the system with respect to an overall MSE criterion is a hard problem. We propose a practical design that combines the polynomial coding in Section II with the codebook design in Section III. The resulting system is illustrated in Fig. 3.

The optimal decoder for the proposed system, in the minimum MSE sense, is

$$
\hat{x} \triangleq E\left[x \mid \boldsymbol{y}_{d}, \boldsymbol{y}_{a}\right]
$$

where $\boldsymbol{y}_{d} \triangleq\left[y_{1}, \ldots, y_{K}\right]^{T}$ and $\boldsymbol{y}_{a} \triangleq\left[y_{K+1}, \ldots, y_{N}\right]^{T}$ are the signals received in the digital and analog parts, respectively. The optimal decoder in (19) is complicated to derive and implement in that it involves integrals and sums that cannot be written out in closed form. We propose to approximate (19) with

$$
\hat{x}=\hat{z}+\hat{e},
$$

where $\hat{z}$ and $\hat{e}$ are estimates of $z$ and $e$, obtained from the analog and the digital part separately.

We next give more details on the digital and analog parts of the proposed HDA architecture and derive the estimates $\hat{z}$ and $\hat{e}$ that we will use in (20). In the digital part, $z=q(x) \in \mathcal{Z}$ is mapped by the function $\boldsymbol{f}(\cdot)$ onto a codeword $c \in \mathcal{C}$ that is sent over the channel $\boldsymbol{H}_{d} \triangleq \operatorname{diag}\left\{\left[h_{1}, \ldots, h_{K}\right]\right\}$. The codebook $\mathcal{C}$ is generated offline by minimizing (13). Since the quantization error will be recovered by the analog part, we need to estimate $z$, instead of $x$. Clearly, for the given mapping $\boldsymbol{f}(\cdot)$, the estimate of $z$ can be obtained by de-mapping $\hat{\boldsymbol{c}}$ (the estimate of $\boldsymbol{c}$ )

$$
\hat{z} \triangleq f^{-1}(\hat{\boldsymbol{c}})
$$

The estimate $\hat{c}$ can be obtained by soft-decoding $c$ and rounding it towards the nearest codeword

$$
\hat{\boldsymbol{c}}=\arg \min _{\boldsymbol{c}_{i} \in \mathcal{C}}\left\|E\left[\boldsymbol{c} \mid \boldsymbol{y}_{d}\right]-\boldsymbol{c}_{i}\right\|^{2}
$$

where

$$
\begin{aligned}
E\left[\boldsymbol{c} \mid \boldsymbol{y}_{d}\right] & =\sum_{i=1}^{M} \boldsymbol{c}_{i} P\left(\boldsymbol{c}_{i} \mid \boldsymbol{y}_{d}\right)=\frac{1}{P\left(\boldsymbol{y}_{d}\right)} \sum_{i=1}^{M} \boldsymbol{c}_{i} P\left(\boldsymbol{c}_{i}\right) P\left(\boldsymbol{y}_{d} \mid \boldsymbol{c}_{i}\right) \\
& =\frac{1}{P\left(\boldsymbol{y}_{d}\right) \sqrt{2 \pi \sigma^{2}}} \sum_{i=1}^{M} \boldsymbol{c}_{i} P\left(z_{i}\right) \exp \left(-\frac{1}{2 \sigma^{2}}\left\|\boldsymbol{y}_{d}-\boldsymbol{H}_{d} \boldsymbol{c}_{i}\right\|^{2}\right) \\
& =\frac{\sum_{i=1}^{M} \exp \left(-\frac{1}{2 \sigma^{2}}\left\|\boldsymbol{y}_{d}-\boldsymbol{H}_{d} \boldsymbol{c}_{i}\right\|^{2}\right) \boldsymbol{c}_{i} P\left(z_{i}\right)}{\sum_{i=1}^{M} \exp \left(-\frac{1}{2 \sigma^{2}}\left\|\boldsymbol{y}_{d}-\boldsymbol{H}_{d} \boldsymbol{c}_{i}\right\|^{2}\right) P\left(z_{i}\right)} .
\end{aligned}
$$

The MMSE estimator (22) is used since we want to obtain the best possible performance given the codes we have designed, even if these codes are not strictly optimal in the MSE sense.

Alternatively, $\hat{\boldsymbol{c}}$ can be taken to be the maximum-a-posteriori (MAP) estimate

$$
\hat{\boldsymbol{c}}=\arg \min _{\boldsymbol{c}_{i} \in \mathcal{C}} P\left(\boldsymbol{y}_{d} \mid \boldsymbol{c}_{i}\right) P\left(\boldsymbol{c}_{i}\right) .
$$

Since we consider a uniformly distributed source and utilize the uniform quantizer, $P\left(\boldsymbol{c}_{i}\right), \forall i$, are equal. The MAP 
estimate of $c$ coincides with the ML estimate

$$
\hat{\boldsymbol{c}}=\arg \min _{\boldsymbol{c}_{i} \in \mathcal{C}}\left\|\boldsymbol{H}_{d} \boldsymbol{c}_{i}-\boldsymbol{y}_{d}\right\|^{2} .
$$

From our simulations, we have observed that there is no visible difference in MSE performance, except that in the low SNR region, the HDA system with the soft decoder (21) performs slightly better than the system with the ML decoder (24).

In the analog part, we employ the polynomial coding of [2] to transmit the quantization error $e$ over the parallel channels with gains $\boldsymbol{H}_{a} \triangleq \operatorname{diag}\left\{\left[h_{K+1}, \ldots, h_{N}\right]\right\}$. For a uniformly distributed source $x \sim \mathcal{U}(-1,1)$ and the uniform quantizer, the quantization error $e$ is uniformly distributed over $[-1 / M, 1 / M]$. Scaling $e$ by a factor of $M$, we have $e^{\prime} \triangleq M e \sim \mathcal{U}(-1,1)$, as the input of the polynomial encoder. Decoding the analog part, we can solve (4) to get the estimate of $e^{\prime}$, denoted as $\hat{e}^{\prime}$. Then, the estimate of $e$ is $\hat{e}=\hat{e}^{\prime} / M$.

Remark 3. The general methodology and transmitter structure proposed here would work for any input signal distributions. We use a uniform source to illustrate the main principles. In order to optimize the system for other input distributions, one needs to know the distributions of the quantized source value and of the quantization errors. Specifically, the codebook design depends on the distribution of the quantized value $z$ and the polynomial coding requires knowledge of the distribution of the quantization error in order to find the corresponding orthogonal polynomials.

\section{Simulation Results}

The MSE performance of different codebooks is shown in Fig. 4 and Fig. 5 for the Rayleigh fading channel and the AWGN channel, respectively. The number of channel uses is assumed to be $N=6$. The quantizer $q(\cdot)$ is the uniform quantizer with $M=4,8,16$ quantization levels. The power constraint per channel use is $\rho=1$. Different types of codes are labeled by $M \times N$. "Optimized Codebook $M \times N$ " is the codebook generated by minimizing (13) for unknown $\boldsymbol{H}$. The "Repetition Codes $M \times 6$ " are the M-PAM symbols repeated over 6 channel uses and "Quaternary Codes $4 \times 6$ " are $\boldsymbol{c}_{1}=[-a-1-a-1-a-1]^{T}, \boldsymbol{c}_{2}=[b-1 b-1 b-1]^{T}, \boldsymbol{c}_{3}=-\boldsymbol{c}_{2}, \boldsymbol{c}_{4}=-\boldsymbol{c}_{1}$, with $a=3 / \sqrt{5}, b=1 / \sqrt{5}$. It can be observed that the "Optimized Codebook $4 \times 6$ ", "Optimized Codebook $8 \times 6$ " and "Optimized Codebook $16 \times 6$ " result in smaller MSEs than other heuristic codes for the same quantization level, not only for the Rayleigh fading channel but also for the AWGN channel. Increasing $M$ from 4 to 8, 16 leads to better performance mainly because with a higher quantization level, the quantization error, which can not be recovered, is smaller. Clearly, a joint optimization of the quantization level, the number of channel uses and the codebook will lead to better performance. However, this is outside the scope of this paper.

A performance comparison of the proposed HDA scheme with pure analog and pure digital schemes is shown in Fig. 6 for $N=6$ parallel Rayleigh fading channels. The following schemes are included in the comparison:

- "Polynomial Coding": polynomial coding (Section II).

- "MRC": maximal ratio combining. The same sample is scaled to fulfill the power constraint and transmitted on all channels. The estimate of the transmit sample is obtained by optimally combining the received signals.

- "Digital Transmission": codebook optimization (Section III). 
- "HDA MRC, $K+K^{\prime \prime}$ : proposed hybrid scheme (Section IV) with $K$ channel uses for the digital part and $K^{\prime}$ channel uses for the analog part and using MRC in the analog part.

- "HDA Polynomial Coding, $K+K^{\prime \prime}$ ": hybrid scheme with $K$ channel uses for the digital part and $K^{\prime}$ channel uses for the analog part, and using polynomial coding in the analog part.

All HDA schemes use the uniform quantizer with $M=4$ quantization levels and the codebooks generated by minimizing (13). The soft decoder (21) is used in the digital part. The index set $\mathcal{I}$ for polynomial coding is chosen to be $\mathcal{I}=\{1,2\}$ for $K^{\prime}=2, \mathcal{I}=\{1,2,5,6\}$ for $K^{\prime}=4$ and $\mathcal{I}=\{1,2,5,6,17,18\}$ for $N=6$, which is suggested in [2].

It can be observed that the HDA scheme outperforms both the digital and analog schemes in the high SNR region and outperforms the analog scheme in the low SNR region. In the low SNR region, the MSE is dominated by the channel distortion incurred by the digital transmission. Since the number of channel uses for digital transmission in the HDA scheme is smaller than in the pure digital scheme, the digital scheme outperforms the HDA scheme. For high SNR, due to the unrecoverable quantization error, the pure digital scheme has an error floor. The HDA scheme can utilize the analog part to compensate the quantization error, which leads to better performance. Note that when the number of the channel uses in the analog part is small, i.e., $K^{\prime}=2$, the HDA scheme with MRC has better performance than the HDA scheme with polynomial coding. The reason for this is that the degrees of the polynomials are too low. By increasing the number of channel uses in the analog part, the HDA scheme "HDA Polynomial Coding, $2+4$ " outperforms the polynomial coding scheme in the high SNR region. This is due to the fact that the quantization error has a smaller amplitude than the source such that the error can be recovered with lower MSE.

Generally, for the HDA schemes, the more channels we assign to the analog part, the better performance we get in the high SNR region, and the worse performance we get in the low SNR region, see Fig. 6. Therefore, the transmission can be adapted according to the SNR: digital transmission in the low SNR region; HDA transmission with more channel uses for the digital part in the medium SNR region (HDA with MRC is preferable, because the MRC decoder is much simpler than the decoder of the polynomial coding); and HDA transmission with more channel uses for the analog part in the high SNR region.

Heuristically, $M$ is not necessary to be large for HDA schemes. The reason is as follows. The quantization error will be recovered by the analog part, so the impact of $M$ on the MSE performance is much weaker than in the digital transmission. Moreover, if $M$ is large, more channel uses should be assigned to the digital part to maintain the performance. The optimization of $M$ is beyond the scope of the paper, which will studied in the future work.

On the AWGN channel, unlike for the Rayleigh fading channel, even with a small number of channel uses in the analog part, the HDA scheme with polynomial coding outperforms the HDA scheme with MRC, see Fig. 7. Except that, similar behavior can be observed as for the Rayleigh fading channel.

\section{CONCLUSIONS}

HDA transceiver architectures can combine the best of the digital and analog world. We have proposed a HDA scheme for low-latency transmission of a single analog value over parallel Rayleigh channels. The scheme outperforms pure digital and analog transmission over a wide range of SNRs. 


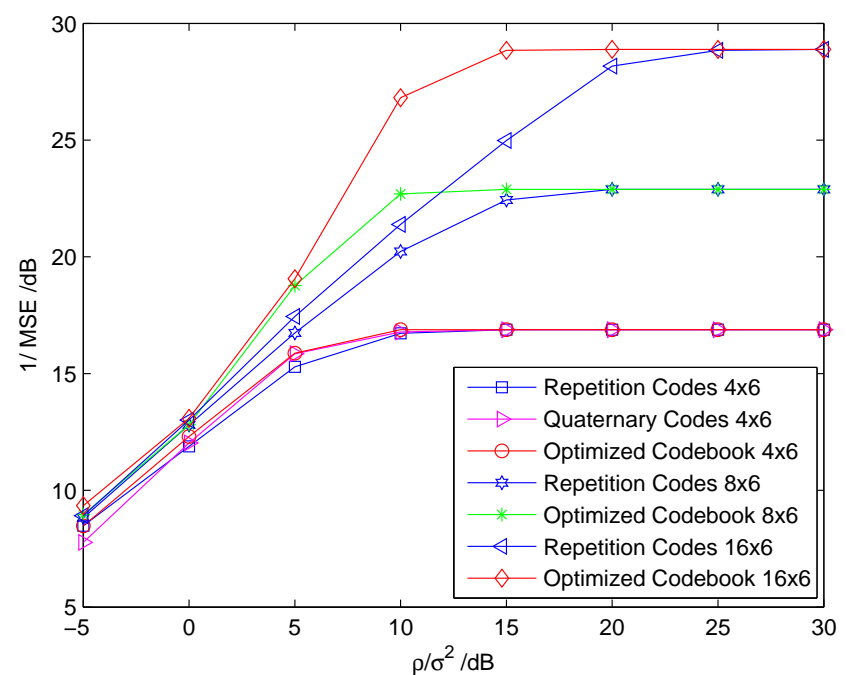

Fig. 4. Codebooks generated by minimizing (13) versus heuristic codebooks. Rayleigh fading channel, $N=6$ channel uses, $\rho=1$.

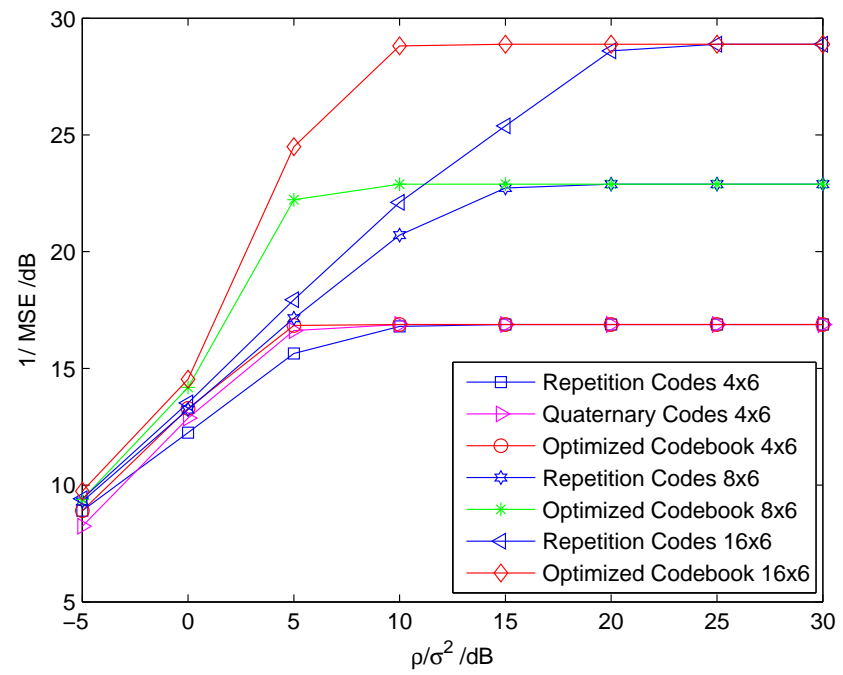

Fig. 5. Codebook generated by minimizing (13) versus heuristic codebooks. AWGN channel, $N=6$ channel uses, $\rho=1$.

\section{REFERENCES}

[1] V.A. Vaishampayan and S.I.R. Costa, "Curves on a sphere, shift-map dynamics, and error control for continuous alphabet sources," IEEE Trans. Inf. Theory, vol. 49, no. 7, pp. 1658 - 1672, jul. 2003.

[2] N. Wernersson, M. Skoglund, and T. Ramstad, "Polynomial based analog source-channel codes," IEEE Trans. on Commun., vol. 57, no. 9, pp. 2600-2606, sep. 2009.

[3] U. Mittal and N. Phamdo, "Hybrid digital-analog (HDA) joint source-channel codes for broadcasting and robust communications," IEEE Trans. Inf. Theory, vol. 48, no. 5, pp. 1082 -1102, may. 2002.

[4] M. Skoglund, N. Phamdo, and F. Alajaji, "Hybrid digital-analog source-channel coding for bandwidth compression/expansion," IEEE Trans. Inf. Theory, vol. 52, no. 8, pp. 3757 -3763, aug. 2006.

[5] "FP7-LOLA project deliverables 2.1 and 2.2 (public)," www.ict-lola.eu.

[6] L. Rade and B. Westergren, Beta Mathematics Handbook, Chartwell-Bratt, Lund, 1989.

[7] J. M. McNamee, “A bibliography on roots of polynomials," J. Comput. Appl. Math, vol. 47, pp. 391 -394, 1993.

[8] N. Farvardin, "A study of vector quantization for noisy channels," IEEE Trans. Inf. Theory, vol. 36, no. 4, pp. 799 -809, jul. 1990. 


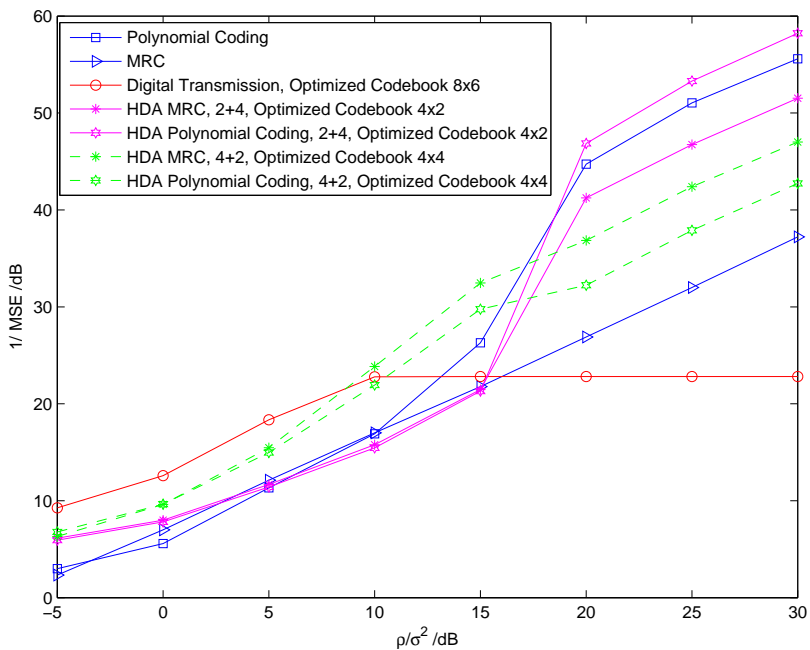

Fig. 6. 1/MSE v.s. $\rho / \sigma^{2}$ for different HDA, analog, digital schemes. Rayleigh fading channel, $N=6$ channel uses, $\rho=1$.

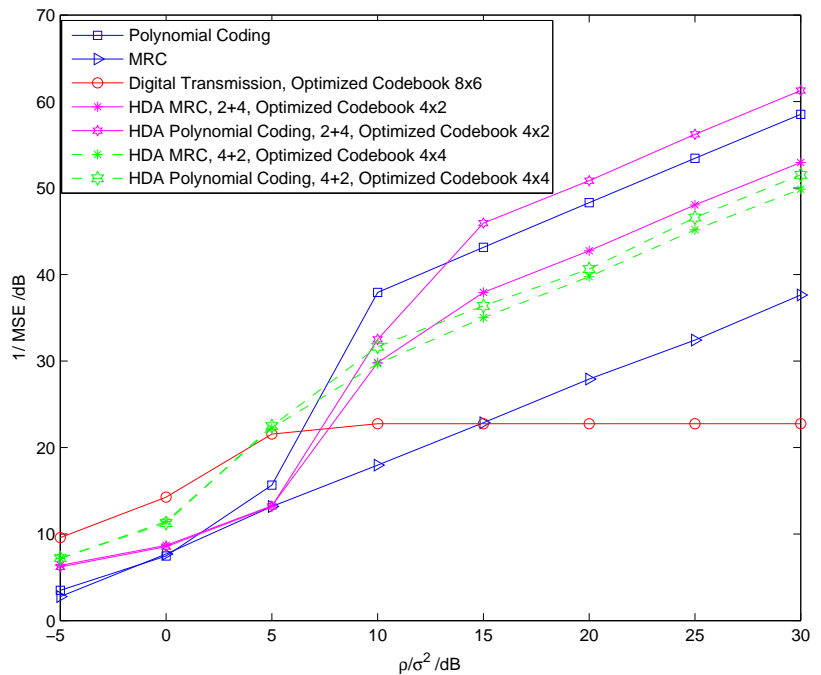

Fig. 7. 1/MSE v.s. $\rho / \sigma^{2}$ for different HDA, analog, digital schemes, AWGN channel, $N=6$ channel uses, $\rho=1$. 